\title{
Faire d'une passion une profession : la place des syndicats d'initiative dans le tourisme français
}

Turning a passion into a profession: the place of tourist offices in French tourism

Julie Manfredini

\section{CpenEdition}

\section{Journals}

Édition électronique

URL : http://journals.openedition.org/tourisme/2327

DOI : 10.4000/tourisme.2327

ISSN : 2492-7503

Éditeur

Éditions touristiques européennes

Référence électronique

Julie Manfredini, « Faire d'une passion une profession : la place des syndicats d'initiative dans le tourisme français », Mondes du Tourisme [En ligne], 16 | 2019, mis en ligne le 01 décembre 2019, consulté le 09 avril 2020. URL : http://journals.openedition.org/tourisme/2327 ; DOI : https://doi.org/ 10.4000/tourisme.2327

Ce document a été généré automatiquement le 9 avril 2020.

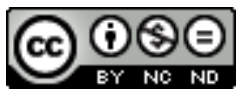

Mondes du tourisme est mis à disposition selon les termes de la licence Creative Commons Attribution - Pas d'Utilisation Commerciale - Pas de Modification 4.0 International. 


\title{
Faire d'une passion une profession : la place des syndicats d'initiative dans le tourisme français
}

Turning a passion into a profession: the place of tourist offices in French tourism

\author{
Julie Manfredini
}

1 Si le fait touristique fait son apparition au début du XIX ${ }^{e}$ siècle avec la multiplication de l'usage des termes tourist et tourism, outre-Manche puis en France, le processus d'industrialisation marque particulièrement la seconde moitié du siècle. Catherine Sicart en rappelle le processus opératoire, comprenant la modernisation des transports, les mutations du système bancaire, les transformations urbaines ainsi que l'apparition d'organismes officiels spécialisés dans le voyage (Sicart, 2008). L'intérêt pour cette activité à fort potentiel économique fit rapidement l'objet d'études économiques, lui conférant de fait un statut (Sicart, 2008) ${ }^{1}$. S'il est difficile de percevoir l'apport économique du tourisme, par manque de données statistiques, cette activité prend suffisamment d'importance pour intéresser de nombreuses catégories socioprofessionnelles, façonnant et consolidant les réseaux touristiques. Si "l'ouverture officielle du chantier scientifique du tourisme» ne date que de 1929 (Sicart, 2008, p.120), la lente professionnalisation des acteurs touristiques se met en place dès la fin du XIX siècle avec l'apparition d'acteurs peu à peu spécialisés dans la mise en tourisme du territoire.

2 En effet, si l'État ne s'intéresse officiellement au tourisme qu'à partir de 1910, avec la mise en place de l'office national du tourisme (ONT) et ce malgré un succès relatif, les syndicats d'initiative (SI) apparaissent en France dès 1889 et construisent rapidement les premiers réseaux touristiques. L'étude proposée ici s'appuie sur notre thèse, intitulée Le rôle des syndicats d'initiative dans la construction de l'identité française de la fin du XIXe siècle aux années $1970^{2}$, rédigée à partir des documents émis par les SI et révélant les différentes personnalités intéressées par le tourisme. Elle reprend la périodisation initiale, de la naissance du premier SI à Grenoble en 1889 - modèle qui sera suivi par des centaines d'autres - aux années 1970, marquées par leur lente disparition au profit 
des offices du tourisme (OT) ${ }^{3}$. À travers l'étude des comptes-rendus des conseils d'administration des SI, de leur correspondance, de leurs bilans de trésorerie, de leur publicité touristique, ainsi que des articles de journaux relatant leurs actions, les premiers constats montrent une forme associative locale innovante, regroupant l'ensemble des professions intéressées par le tourisme, selon le modèle des sociétés de développement suisses. Dans un premier temps dévolus à l'accueil et au renseignement des touristes, les SI accomplissent la mise en tourisme du territoire sous de multiples formes et valorisent le patrimoine local, qu'il soit matériel ou immatériel. Comme l'explique Nabila Oulebsir, les SI, très tôt considérés comme les bases du tourisme réceptif, sont devenus des "agence de marketing" (2011, p. 127), dont les actions dynamiques et variées étaient connues des réseaux touristiques, reconnues par l'État, mais peu soutenues. Les difficultés quotidiennes, accompagnées d'un appui national insuffisant, ont fait des SI les parents pauvres du tourisme français. Néanmoins, ils ont joué un rôle essentiel dans la mise en tourisme du territoire et dans la reconnaissance des professionnels du tourisme pendant quatre-vingt ans. Les SI mentionnés dans cet article concernent l'ensemble du territoire français, révélant des pratiques communes. Toutefois, à plusieurs occasions, nous nous appuyons sur certains SI particuliers, comme celui de Grenoble qui, par sa naissance et le modèle qu'il constitue, est particulièrement cité ici. De même, le SI de Provence et celui de Saint-Brieuc représentent des exemples solides du processus de création d'une identité territoriale forte, dont les traces sont encore visibles aujourd'hui à travers les marques «Provence» et «Bretagne». Néanmoins, c'est la multiplicité des exemples pris sur l'ensemble du territoire métropolitain français qui révèle l'ampleur de cet engagement des élites culturelles locales.

3 À travers cet article, il s'agit de raconter l'histoire de ces bénévoles passionnés par leur ville, leur région ou leur patrie, de 1889 aux années 1970, et d'observer ce «monde multiforme» (Levati, 2009) qui place le tourisme au cœur de ses préoccupations. L'écriture du voyage, sous toutes ses formes, est «l'un des fondements de l'industrie touristique» (Hoerner et Sicart, 2003, p. 82) auquel les SI français contribuent grandement par leur publicité, activité qui représente les deux tiers de leur budget. En effet, à travers les guides, dépliants puis affiches - héritiers des livres de voyages -, les SI dressent le portrait de la destination touristique. Cette «image culturelle $»^{4}$, attendue par les touristes, est issue de l'héritage culturel des acteurs touristiques et reflète leurs connaissances et leurs passions, constitutives de leur identité. Ainsi, en écrivant l'histoire non exhaustive de ces acteurs, passionnés et avant-gardistes, moteurs de ces associations, nous dressons le portrait de ces organismes privés et dynamiques, dotés d'une grande capacité d'adaptation, inscrits au cœur du processus touristique français ainsi que des émotions au centre de cette construction des destinations touristiques.

\section{Le rôle de la bourgeoisie culturelle au sein du SI}

\section{Composition d'un SI}

4 D'après les sources utilisées, les SI s'inscrivent au cœur des réseaux touristiques français notamment grâce au cumul des fonctions, pratique courante et recherchée. De nombreux corps de métiers sont suffisamment intéressés par le tourisme pour être régulièrement présents dans la liste des membres ( $c f$. illustration 1), même si notre 
étude ne peut être exhaustive ${ }^{5}$. D'ailleurs, nous verrons combien la composition des SI illustre les transformations de la société et de son époque. Cependant, si certains points communs peuvent être relevés, aucun SI ne se ressemble, chacun préférant définir son identité en fonction des besoins de sa communauté. Comme le montre l'illustration 2, différents acteurs ont, tour à tour, été au cœur des SI. Dans un premier temps, les membres de la noblesse et de la bourgeoisie traditionnelle jouent un rôle important, tant dans la formation des syndicats que dans le financement de leurs actions. La réputation de certains noms offre un appui non négligeable, même si ces acteurs deviennent rapidement membres honoraires, délaissant les hautes fonctions une fois le plaisir de l'innovation disparu. Ils laissent ainsi la place aux élites modernes, soucieuses de s'investir tant dans la politique culturelle locale que sur le territoire alentour, qui vont diriger les SI pendant soixante ans avant que la marchandisation du tourisme et des loisirs ne fasse la part belle aux professionnels du tourisme.

Illustration 1. Composition des SI

\begin{tabular}{|c|c|}
\hline Nom du SI & Membres du bureau - leur fonction- leur métier ou position sociale \\
\hline $\begin{array}{l}\text { SI de Nancy et des } \\
\text { Vosges } \\
\text { En } 1903\end{array}$ & $\begin{array}{l}\text { H. Gutton -président- architecte et administrateur du tramway } \\
\text { Ph. Berger - vice-président - conseiller général de Giromagny } \\
\text { A. Parisot - vice-président - administrateur de la société des Thermes de } \\
\text { Plombières } \\
\text { J. Garnier - vice-président - industriel } \\
\text { Perrigot-Masure - secrétaire- président AC Vosgien }\end{array}$ \\
\hline $\begin{array}{l}\text { SI de Lyon } \\
\text { En } 1910\end{array}$ & $\begin{array}{l}\text { A. Rivoire - président - membre de la chambre de commerce et président } \\
\text { honoraire du syndicat des horticulteurs } \\
\text { A. Millet - vice-président - ancien juge au tribunal de commerce } \\
\text { F. Quinnon - vice-président - délégué au TCF }\end{array}$ \\
\hline $\begin{array}{l}\text { SI de Bollène } \\
\text { En } 1930\end{array}$ & $\begin{array}{l}\text { E. Gachet - président- ............ } \\
\text { L'abbé Clavel -vice-président - religieux } \\
\text { Commandant Dugloud - vice-président - militaire } \\
\text { Escoffier - trésorier - banquier } \\
\text { J. Raoux - secrétaire ou archiviste - artiste } \\
\text { Auriac - secrétaire ou archiviste - imprimeur }\end{array}$ \\
\hline $\begin{array}{l}\text { SI de Fontainebleau } \\
\text { En } 1935\end{array}$ & $\begin{array}{l}\text { É. Sinturel - président - inspecteur principal (forêt de Fontainebleau) } \\
\text { R. Galant - vice-président - artiste } \\
\text { Capitaine Aubertin - vice-président - militaire } \\
\text { C. Ballen de Guzman - secrétaire - notable équatorien installé à } \\
\text { Fontainebleau }\end{array}$ \\
\hline
\end{tabular}


Illustration 2. Les catégories sociales des membres des SI et leur évolution

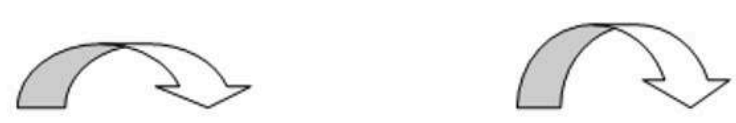

\begin{tabular}{|l|}
\hline $\begin{array}{c}\text { Noblesse et bourgeoisie } \\
\text { traditionnelle }\end{array}$ \\
Nombreuses à la fin du \\
XIXe siècle. Fondatrices des \\
SI ou membres honoraires \\
dont les fonds sont \\
recherchéspourfinanorles \\
activités dusl.
\end{tabular}

\begin{tabular}{|l|}
\hline \multicolumn{2}{|c|}{ Élites modernes } \\
Présentes dès la fin du \\
XIXe siècle, rapidement \\
devenues la plus active de \\
toutesles catégories présentes. \\
Elles exercent aux plus hautes \\
fonctions du sl et cumulent des \\
mandats auprès d'autres
\end{tabular}

Professionnels du tourisme

Déjà présents auXIX`e siède. Catégorie qui atteint les plus hautes fonctions après 1960. Familiers de la marchandisation du tourisme, ils accélèrent la perte d'autonomie des SI.

5 Ces membres, quelle que soit leur origine sociale, ont de nombreux points communs, dont leur dynamisme, leur intérêt pour le tourisme, leurs passions culturelles et une grande capacité d'engagement en faveur de leur territoire. Ensemble, ils participent à la construction d'une culture commune de référence. L'exemple du SI de Grenoble, étudié dans la thèse de Bertrand Larique (2006, p.147), nous éclaire quant au recrutement des membres du SI et révèle un élargissement du recrutement au début du $\mathrm{XX}^{\mathrm{e}}$ siècle. En effet, comme l'illustre le graphique 1, le SI grenoblois recrutait en 1899 principalement au sein de la ville : près de $86 \%$ des membres du SI résidaient en ville, seules 59 personnes ne provenaient pas de la capitale du Dauphiné. Au début du siècle, les SI tentent de consolider leur assise régionale en recrutant leurs membres sur un territoire plus vaste. De fait, en 1910, le SI de Grenoble compte 664 adhérents dont 224 ne résident pas dans la ville mais sont issus d'autres départements comme la Savoie, la Haute-Savoie, la Drôme et les Hautes-Alpes (cf. graphique 2).

Graphique 1. Composition des SI, l'exemple Grenoblois en 1889

\section{$86 \%$ \\ habitant à Grenoble}

$14 \%$ habitant hors Grenoble

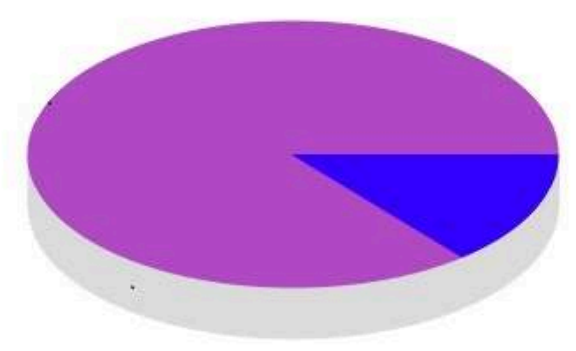




\section{$66 \%$ \\ habitant à Grenoble \\ $34 \%$ \\ habitant hors Grenoble}

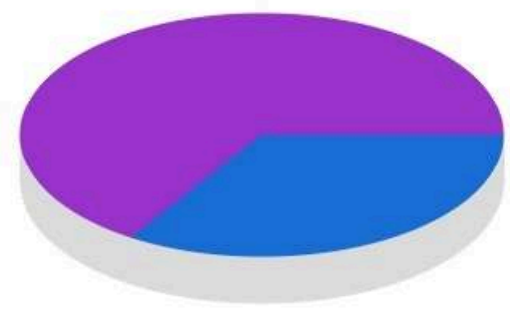

\section{Affirmation des élites culturelles ${ }^{6}$}

6 Si certains corps de métiers sont très intéressés par les retombées économiques du tourisme, comme les hôteliers, les restaurateurs ou les commerçants (dont les épiciers, les cafetiers, les limonadiers ou les boulangers), ce ne sont pourtant pas eux les plus actifs. En effet, tout comme les grands propriétaires rentiers présents parmi les pionniers, les professionnels intéressés par le tourisme sont, dans un premier temps, soit relégués au titre de membres d'honneur, soit fondus dans la masse des adhérents. Ce sont les élites modernes, comme les avocats, les industriels, les membres des chambres de commerce ou les publicistes qui semblent devenir les moteurs de cette association touristique. Cette situation, accentuée par un manque d'engagement de la part des professionnels du tourisme, est régulièrement critiquée et dénoncée par l'ensemble des SI alors que ces professionnels sont les premiers bénéficiaires des retombées. Toutefois, certaines personnalités locales actives marquent les lieux et contribuent au développement de leur ville. Ce fut le cas d'Henry de Jouvenel, sénateur de Corrèze (1921 et 1933), animateur des État généraux du tourisme en 1913. Son action a permis la naissance de plusieurs SI, dont cinq en Corrèze et dix en Dordogne, d'où le surnom de « champion du tourisme » qui lui est attribué (Manigand, 2000, p. 164). Sur le long terme, leurs actions parviennent à créer une mise en tourisme efficace du territoire, justifiant une récompense pour certains membres. Certains se sont vus attribuer la légion d'honneur, comme Paul Arnal, fondateur du Club Cévenol, qualifié d'« apôtre du tourisme dans les Cévennes et les Causses » (cf. illustration 3). 
Illustration 3. Paul Arnal, fondateur du club cévenol

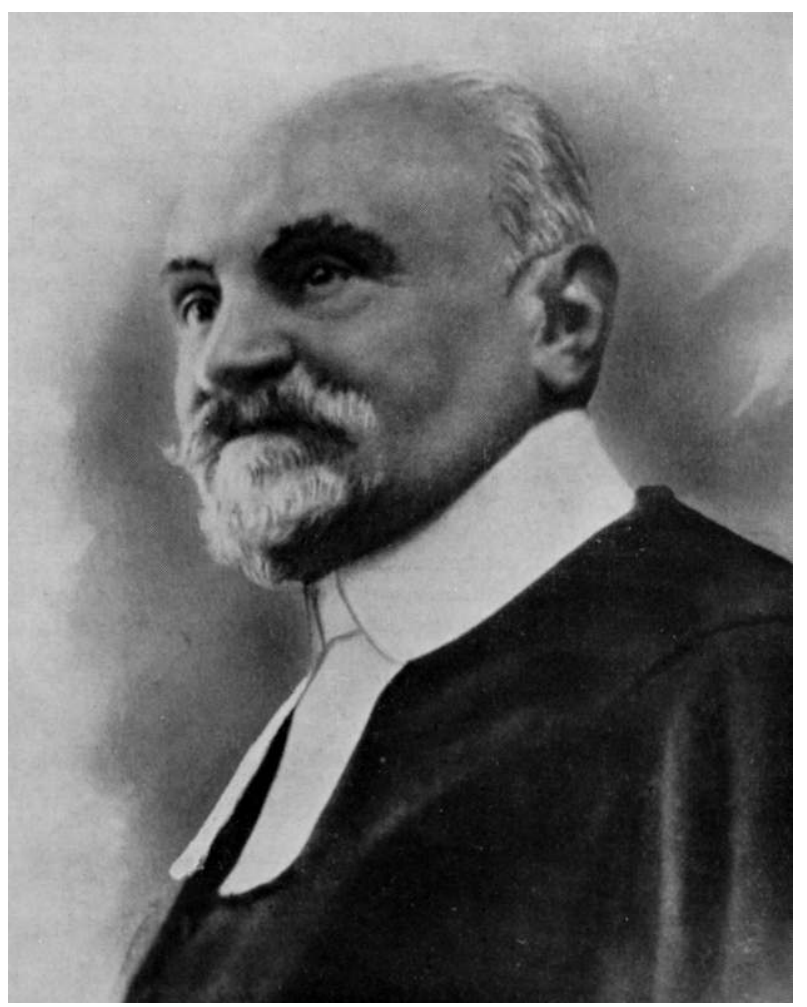

Musée des vallées cévenoles, coll. Particulière Catherine Poudevigne

7 Comme l'explique Olivier Poujol, cette récompense, reçue en 1929, est venue célébrer les mérites de ce passionné, très actif dans la promotion du tourisme français ${ }^{7}$. D'autres ont reçu une récompense spécialement pensée et créée par les acteurs du tourisme français. Entre 1949 et $1963^{8}$, la croix du mérite touristique a mis à l'honneur l'œuvre de nombreuses personnalités, grâce à l'initiative de Marcel Bellon9, président du SI du Queyras. Lui-même a reçu cette décoration en juin $1950^{10}$. Enfin, certaines villes ont pris le parti de récompenser ces entrepreneurs du tourisme, qui ont marqué le développement et la promotion de leur territoire, par l'attribution de leur nom à un lieu ou un objet. Ce fut le cas à Lyon, où le nom d'Achille Lignon, président d'honneur et fondateur du SI, fut inséré au cœur de la toponymie de la ville avec la création d'un quai éponyme ${ }^{11}$. Ces « entrepreneurs du loisir » (Réau, 2011, p. 17) usent de leur passion et de leurs réseaux pour compléter et diversifier l'offre touristique.

\section{Des acteurs parfois très influents}

Parmi les acteurs les plus influents, de par leurs qualités et leurs réseaux, les avocats ont joué un rôle important dans les débuts des SI, allant jusqu'à s'imposer dans les plus hautes fonctions ou se faisant les fondateurs de ces associations novatrices. Leurs intérêts personnels ont rejoint leur passion pour le tourisme, faisant de ces grands orateurs des acteurs indispensables. Armand Chabrand, président du SI de Grenoble en 1904, illustre la position de ce groupe ${ }^{12}$. Cet alpiniste chevronné présidait déjà la Société des touristes en Dauphiné, avant de prendre la direction du SI et était l'auteur de nombreux ouvrages sur les coutumes dauphinoises et le tourisme et de guides touristiques. Sans surprise, les médecins jouent également de leur influence, profitant 
de l'engouement pour le thermalisme et œuvrant au développement des stations. Quant aux milieux journalistiques, présents et impliqués, ils n'ont cessé de s'imposer. Rédacteurs en chef, journalistes et publicistes veillent à la promotion touristique de leur ville, comme le fit Léon Goulette à Nancy. Journaliste de L'Est Républicain et fondateur du SI de Nancy et des Vosges en septembre 1903, il a contribué au développement du thermalisme vosgien, devenant un acteur indispensable du développement économique, culturel et touristique de la région (Roth, 2004).

Bien que pionniers en matière de tourisme, les SI n'ont pas non plus été à contrecourant de leur époque. Les femmes n'y ont pas de réelle place, si ce n'est comme accompagnatrices de leurs maris. Contrairement à certaines associations professionnelles et caritatives qui, dès la fin du XIX e siècle, offrent aux femmes uneidentité publique alors qu'elles sont exclues des suffrages, tel n'est pas le cas des SI (Fraisse et Perrot, 2002). Au sein de notre corpus de documents, seules deux femmes atteignent des postes élevés: Jane Raoux au SI de Bollène dans les années 1930 et la comtesse d'Agay à la tête du SI d'Agay. La première, artiste peintre et fille du maréchal forgeron, est mentionnée régulièrement comme secrétaire adjointe ou archiviste jusqu'en $1946^{13}$. Cette année-là, elle préside les réunions du conseil d'administration, remplaçant momentanément le président, tout en étant accompagnée de trois vice-présidents quand habituellement un ou deux suffisent. La seconde, sœur d'Antoine de Saint-Exupéry, lutte sans relâche en 1949 contre le SI de Saint-Raphaël qui veut absorber celui d'Agay, jugé trop proche et contreproductif au sein de la politique touristique locale ${ }^{14}$. En général, les femmes sont plutôt mentionnées comme personnel d'accueil, comme c'est le cas pour le SI d'Albertville en $1924^{15}$. La plupart se perdent dans la masse des adhérents, seule une présence figurative leur est proposée. Elles reçoivent des présents et font des visites touristiques programmées lors des déplacements, pendant que leurs homologues masculins discutent autour de la table. Néanmoins, la situation progresse peu à peu et certaines deviennent déléguées du SI, comme le montre l'exemple du SI du Queyras en 1955 où quatre des cinq délégués qui œuvrent dans les territoires voisins sont des femmes, notamment à Nice, Lyon et Toulon ${ }^{16}$. Par ailleurs, certaines sont récompensées pour leur action en faveur du tourisme au même titre que leurs collègues masculins. Ce fut le cas pour Odette Chevalier dont les trente années au service de la fédération de Franche-Comté-Jura furent célébrées par l'attribution de la croix de chevalier du mérite touristique ${ }^{17}$. Toutefois, dans les années 1960, il semble que les femmes œuvrant à des postes élevés demeurent des exceptions; elles sont plutôt cantonnées au rôle d'hôtesse au sein des bureaux, comme à Grenoble où les robes bleues sont devenues leur signe distinctif ${ }^{18}$.

\section{Naissance d'une passion ${ }^{19}$, prémices d'une profession}

\section{Un groupe qui partage les mêmes objectifs}

10 S'il existe, à la fin du $\mathrm{xIX}^{\mathrm{e}}$ siècle, des syndicats professionnels ou des associations spécialisées dans un domaine œuvrant à la défense d'un métier ou d'un terroir, la naissance des SI relève d'un tout autre objectif. Fondés sur le modèle des sociétés de développement suisses, apparues dans les années 1880, les syndicats d'initiative 
entendent rassembler les professionnels et particuliers intéressés par le tourisme, directement ou indirectement. Ainsi sont nés ces «groupes de citoyens, désintéressés qui, chacun dans [leur] localité mettent en œuvre, proportionnellement à leurs ressources, leurs moyens propres à perfectionner les conditions de séjour de leurs hôtes $»^{20}$. Unis dans l'effort pour accomplir une œuvre nationale, les membres étaient emplis d'idéaux et se surnommaient les " apôtres du tourisme ", conscients de leur rôle dans un système touristique fragile ${ }^{21}$. En effet, l'État se préoccupe peu du tourisme avant la création de l'office national du tourisme (1910) et l'intérêt de quelques personnalités comme Étienne Clémentel, fondateur du groupe du tourisme à la Chambre des députés en 1912 (Larique, 2007) ${ }^{22}$. Si, avant la Première Guerre mondiale, la France se dote au niveau national d'un « tourisme à trois têtes », composé de l'Union nationale des associations de tourisme (UNAT), de l'Union des fédérations des SI (UFSI) et de l'Office national du tourisme (ONT), les SI locaux et régionaux sont régulièrement salués pour leurs actions. Mais il faut attendre 1935 pour voir l'apparition d'un Commissariat général du tourisme (Larique, 2006) institué dans le cadre de la montée des périls et de l'instrumentalisation des acteurs du tourisme en faveur de la nation. Ainsi, si les SI sont «au cœur du système de construction du tourisme", ce sont également des « institutions difficiles à cerner » (Bertho-Lavenir, 1999, p. 259).

Aussi, dans le cadre de la lente reconnaissance des SI et de l'appui fragile dont ils bénéficient au niveau national, le président du SI est un personnage emblématique, entrepreneur du tourisme et souvent avant-gardiste, dont la personnalité active marque la structure et les réseaux auxquels il participe. Décrits la plupart du temps comme des personnalités souriantes et aimables, le côté provocateur n'est pas non plus pour déplaire et devient parfois une qualité pour être la figure de proue du SI. Ces qualités le font réélire notamment lorsque le " courage, l'expérience [et] l'amabilité ${ }^{23}$ sont présents, parce qu'un bon président est un homme qui reste longtemps à la tête de son syndicat et qui en marque l'esprit général. D'ailleurs, les plus influents au sein des réseaux touristiques cumulent de nombreuses fonctions sur plusieurs années, leur permettant une visibilité touristique d'un point de vue économique, culturel et territorial. Le cas de Maître Louis Porte illustre cette influence, tant au sein du SI de Grenoble, qu'il administre pendant vingt ans avant d'en être le président, qu'au sein d'autres organismes tels que le comité d'organisation des JO d'hiver de 1968, les Amis de l'université de Grenoble ou la Société dauphinoise de secours de montagne (cf. tableau 1).

Tableau 1. L'influence des présidents des SI

\begin{tabular}{|l|l|l|}
\hline Nom & SI & Éléments biographiques \\
\hline
\end{tabular}




\begin{tabular}{|c|c|c|}
\hline $\begin{array}{l}\text { Maître Louis } \\
\text { Porte } \\
\text { (décédé en } \\
\text { 1965) } \\
\text { Élu en } 1951 \\
\text { Réélu en } 1960 \\
\text { et } 1964\end{array}$ & Grenoble & $\begin{array}{l}\text { - avocat à la cour d'appel de Grenoble } \\
\text { - administre le SI pendant vingt ans avant d'accéder à la } \\
\text { présidence } \\
\text { - administrateur du comité d'organisation des JO d'hiver } \\
\text { (1968) } \\
\text { - président de la Société dauphinoise de secours de } \\
\text { montagne } \\
\text { - membre de l'administration des Amis de l'université de } \\
\text { Grenoble. } \\
\text { - officier du mérite touristique, de la légion d'honneur et du } \\
\text { mérite national } \\
\text { - Chevalier des Palmes académiques et décoré de la croix de } \\
\text { guerre (14-18) }\end{array}$ \\
\hline $\begin{array}{l}\text { Pierre } \\
\text { Gravier } \\
\text { Élu en } 1934 \\
\text { Réélu en } 1944\end{array}$ & $\begin{array}{l}\text { Briançonnais- } \\
\text { Pelvoux }\end{array}$ & $\begin{array}{l}\text { - fondateur et président du club de hockey de Briançon } \\
\text { (1934). } \\
\text { - président du comité du tourisme départemental des } \\
\text { Hautes-Alpes } \\
\text { - membre du Comité régional du tourisme de Grenoble } \\
\text { - président de l'office de tourisme des Hautes-Alpes (Gap) en } \\
1954 \\
\text { - vice-président de la chambre de commerce de Gap (1954) } \\
\text { - président du syndicat des hôteliers de Cannes et membre } \\
\text { de l'administration du SI de Cannes }\end{array}$ \\
\hline $\begin{array}{l}\text { Jean Durand } \\
\text { En place de } \\
1947 \text { à } 1949\end{array}$ & Nîmes & $\begin{array}{l}\text { - s'occupe du SI de Nîmes depuis } 1920 \text { (secrétaire général } \\
\text { pendant quinze ans) } \\
\text { - cumule les vice-présidences du Club Cévenol, du comité } \\
\text { des fêtes de Nîmes et de la fédération des SI de Provence } \\
\text { - préside l'union départementale des SI du Gard } \\
\text { - membre du CRT de Marseille }\end{array}$ \\
\hline
\end{tabular}

\section{Partage et création d'une culture commune}

Les membres du bureau du SI sont ceux qui, avec le président, œuvrent le plus à la promotion de leur structure et de sa politique touristique. Leur étude permet de comprendre le degré de mobilisation des intellectuels présents dans les SI, leurs prédilections et les répercussions tant sur le territoire que sur la société locale. Ils placent peu à peu le loisir au centre de leurs préoccupations individuelles dans l'intérêt de la société (Corbin, 1995). En effet, ces personnalités locales sont animées d'une passion pour leur territoire, sa promotion et le développement touristique bien avant que les notions de vacances et de loisirs et que la propagande touristique ne soient intégrées au quotidien des Français. Il semble qu'un fort sentiment patriotique soit partagé par ces notabilités culturelles et rejaillisse, par leurs actions, sur l'identité de leur territoire (Goujon, 1989). En effet, certains perçoivent leur engagement au sein du SI comme un acte patriotique, finalement peu éloigné de l'engagement militaire, voire comme le prolongement d'un tel engagement. Ainsi, il n'est pas étonnant d'y retrouver d'anciens combattants ou des militaires de carrière, comme le commandant Alfred Trouillet à Bollène ${ }^{24}$. Engagé volontaire depuis ses 18 ans, il a participé aux deux conflits mondiaux et obtenu la croix de guerre à deux reprises; il s'est intéressé au SI 
de sa ville dès 1939. Si cette couverture était idéale en temps de guerre pour superviser certains actes de résistance, le commandant a poursuivi son engagement civique et culturel au sein du SI en le présidant à partir de 1946. Les membres des SI œuvrent inlassablement à la conservation et à la promotion de l'héritage historique de leur territoire local, établissant dès que possible des parallèles avec l'histoire nationale, inaugurant des musées comme celui de l'archerie à Crépy-en-Valois, instaurant des festivités comme la foire de Dijon, apposant des plaques commémoratives ou organisant des conférences sur le tourisme régional, accompagnées de films et de causeries à destination des élites locales. Ainsi, patrimoines matériel et immatériel sont à l'honneur dans la politique des SI, socle solide illustré par l'émergence du patrimoine gastronomique par exemple, où repas de célébration, apéritifs et congrès commémorent les spécialités culinaires locales, forgeant la réputation des terroirs gastronomiques français.

Cette implication dans la défense, la préservation et la promotion du patrimoine local, et sa concrétisation par des rendez-vous touristiques, est le résultat d'une passion dévorante de ces élites culturelles pour leur territoire et la Culture. Chacun met ainsi ses expériences et ses compétences au service de la promotion publicitaire du territoire. C'est le cas de Paul Ruat et de Paul Testartqui, avec leurs cartes postales, se font les vecteurs des symboles provençaux pour le premier et de la modernisation du territoire d'Épinal pour le second. D'autres mettent leur plume au service des SI, comme Auguste Giry, journaliste et membre du SI de Provence, auteur d'un rapport détaillé intitulé $\mathrm{Du}$ SI de Provence : l'action des SI (1917) et de plusieurs écrits sur l'identité provençale ${ }^{25}$. Passionnées par la culture et l'histoire de leur localité, ces personnalités se donnent les moyens de la faire connaître à travers les guides locaux ou les journaux qu'ils créent parfois spécialement dans ce but. Ce fut le cas d'Octave-Louis Aubert, fondateur du SI de Saint-Brieuc en 1907 et délégué de la fédération des SI de Bretagne ${ }^{26}$. Jusqu'en 1949, ce publiciste, éditeur et historien s'est impliqué dans la promotion de ce territoire, célébré par une multitude d'initiatives dont la création de la revue Bretagne touristique (1922-1939) (Pallier, 1996). Enfin, la valorisation de leur territoire et de son identité est également visible à travers certains événements dont les exemples sont nombreux: de Jules Lafon et la Paulée de Meursault à Jean Héritier et le salon international des santons en Arles, organisé dès 1958 et encore célébré de nos jours. Les personnalités les plus dynamiques au sein des SI montrent donc un attachement particulier à leur ville et aux territoires alentours. Ils se reconnaissent à leur position sociale élevée, à la reconnaissance qui les entoure de par leurs actions et à leur implication, ainsi qu'à leur place au sein des réseaux culturels et touristiques - même si ceux-ci s'entrecroisent de plus en plus. De fait, ces élites culturelles renforcent l'image du tourisme et de ses enjeux, faisant de ce domaine un impératif économique et culturel pour chaque territoire en voie de développement.

\section{Disparaître pour perdurer}

14 Malgré leurs difficultés quotidiennes, les SI ont perduré pendant quatre-vingt ans, devenant le socle solide et indispensable du tourisme français. Toutefois, dès 1965, dans un bilan général, le constat est sans appel et annonce la disparition progressive des SI : «Le temps des bénévoles est révolu, celui des mécènes aussi ». Les SI ont régulièrement réfléchi à de possibles alternatives afin de s'autofinancer, avançant l'idée d'équité afin que chaque acteur touristique cotise proportionnellement à ses 
intérêts, comme le suggérait Pierre Defert, ou la mise en place d'une taxe sur les plusvalues touristiques évoquée par le Docteur Maulini ${ }^{28}$. Néanmoins, ces propositions sont restées incomprises et les SI, déjà fragiles, se sont progressivement essoufflés. L'idéal aurait été d'intégrer le budget des SI au futur plan économique de l'État comme au sein du budget communal, mais le statut d'origine des SI rendait impossible cette solution.

D'où l'apparition des conventions, sortes de contrats passés entre les municipalités et leur SI, formalisant une situation déjà existante. Dès lors, le SI recevait un financement municipal fixe et régulier pour les frais de chauffage, d'éclairage, de poste et le salaire des personnels. En contrepartie, le SI s'accommodait d'une perte d'autonomie et effectuait une mission de service public, à sa seule charge financière. Par conséquent, le fonctionnement du syndicat évolue puisqu'il doit désormais rendre des comptes, ce qui va à l'encontre de ses principes originels, créant des tensions et des déceptions. Dès lors, par exemple, «le SI s'engage à soumettre à l'agrément du maire des candidats à la fonction de directeur, en cas de nécessité de pourvoir à cet emploi », transformant peu à peu le SI en organisme municipal ${ }^{29}$.

\section{III. Évolutions du tourisme et des territoires, le rôle des élites culturelles}

\section{Développer les espaces et les temps culturels dans la ville}

16 La multiplication des compétences dévolues aux SI leur a permis d'élaborer une offre culturelle étendue. Ils ont contribué à la création de lieux culturels renommés, par simple proposition ou par financement, comme des musées ou des théâtres. Ce fut le cas à Marseille où le futur fondateur de l'office du tourisme, Marius Dubois, est également l'un des acteurs ayant œuvré à la création d'un bâtiment au parc chanot transformé à son initiative en Musée du Vieux-Marseille ${ }^{30}$. De même, ce personnage public est à l'initiative de plusieurs événements de grande ampleur, comme la célébration du $25^{\mathrm{e}}$ centenaire de la ville en 1899, l'Exposition coloniale de 1906 ou celle de l'électricité de 1908. L'ensemble des SI, puis des offices du tourisme, proposent, organisent et exposent l'identité de leur territoire lors d'événements culturels spécifiques, comme les salons et les festivals, dont la renommée s'inscrit durablement dans l'identité urbaine, profitant à la fois aux touristes et aux habitants. Cette implication culturelle au sein de l'espace urbain s'inscrit dans un objectif plus général, celui de développer cet espace pour le rendre plus accessible et plus lisible, pour ainsi améliorer le tourisme. D'où la contribution de certains architectes aux SI, pour qui la modernité passe par une transformation de l'espace urbain. Henri Gutton, à Nancy, a mis cette idée en pratique à travers sa participation à l'embellissement et au développement de la cité durant sa présidence du SI dès 1903, avec son engagement en faveur de l'Art nouveau et du tramway ${ }^{31}$. Il a ainsi contribué à moderniser les transports à Nancy et dans les Vosges par le développement des réseaux.

La Seconde Guerre mondiale et la période de reconstruction qui s'ensuit accélèrent cet engagement urbain pris par les SI. En effet, tout en souhaitant redémarrer l'activité touristique de leur ville et proposer une nouvelle offre en relation avec le tourisme de masse qui se profile dans les années 1950, ces structures poursuivent dans un premier temps leur engagement patriotique. Ainsi, avant même la fin du conflit, certains SI se penchent sur l'élaboration d'un nouveau plan d'urbanisme pour leur cité, comme ce fut 
le cas à Bollène. Dès 1941, le SI élabore des plans pour sa ville, notamment pour le square de la place de la bascule, répondant à la demande faite par la mairie ${ }^{32}$. Ces démarches urbanistiques se poursuivent après la guerre, avec des propositions pour conserver l'église du pont et assainir certains quartiers de la ville, dont celui du Puy ${ }^{33}$. Les SI repositionnent aussi leur local au cœur de l'espace touristique de la ville, veillant à ce qu'une meilleure accessibilité puisse faire converger les touristes jusqu'à ce point. Le développement du réseau routier, dans son ensemble, en offrant une meilleure accessibilité, incite les acteurs locaux à moderniser leur politique touristique, à améliorer la préservation et la mise en valeur de leurs monuments historiques (Rauch, 2001). Cela engendre parfois de vives tensions, comme à Bollène pour la préservation de la statue de Pasteur créée par Félix Charpentier ${ }^{34}$. Enfin, les SI insistent sur l'importance d'une politique d'hygiène efficace, accompagnée de l'embellissement des entrées de ville, premier espace visible par les visiteurs. Toutefois, ces nombreuses missions ont été reprises par les municipalités qui ont par la suite souhaité les déléguer aux nouveaux OT, sous leur direction, dans les années 1960-1970.

\section{Le tourisme, une passion en devenir}

Le socle solide sur lequel repose l'activité touristique, en France, est fondé sur une prise de conscience essentielle de la part des acteurs les plus haut placés. Dès la fin du XIX ${ }^{\mathrm{e}}$ siècle, et ce malgré l'absence d'une organisation nationale des SI, ces associations ont tissé de solides réseaux touristiques, entre eux ainsi qu'avec toutes les organisations publiques ou privées intéressées par le tourisme, comme les chambres d'industrie ou les compagnies de cars ainsi que les associations de tourisme comme le Touring Club de France (TCF) ou l'Automobile Club de France (ACF) (Bertho-Lavenir, 1999). Il s'agissait d'établir un esprit de solidarité, sorte de « loyale camaraderie $»^{35}$ dont Léon Auscher et Edmond Chaix se faisaient les promoteurs. On retrouvait d'ailleurs l'ensemble de ces organismes au sein du local du SI, sorte d'espace centralisateur. Par son action, le TCF a d'ailleurs contribué au développement des SI en leur donnant "un élan nouveau " (Bertho-Lavenir, 1996, p. 59), en fédérant les initiatives des élites intellectuelles et sociales locales. Les liens entretenus avec les SI entrent dans la stratégie mise en place par le TCF pour « développer le tourisme sous toutes ses formes " tout en obtenant une reconnaissance officielle pour ses actions (Réau, 2011, p. 34).

Le cumul des fonctions des présidents et des membres du bureau de chaque SI illustre également cette volonté de consolider les réseaux. Ce cumul des fonctions était alors conçu comme une façon de garantir une meilleure continuité des politiques. Le président du SI de Vichy, Robert Mathieu ${ }^{36}$, qui a cumulé les présidences du syndicat des hôteliers (1945) et de la fédération hôtelière de l'Allier (1945), en est un exemple. De même, en 1965, il fut à la fois membre du conseil municipal et du comité des fêtes. Toutefois, si le rôle de certains présidents dans les grandes instances touristiques n'est plus à démontrer, offrant au SI une influence locale et nationale, il est vrai que cette stratégie a ralenti l'évolution des politiques touristiques ainsi que le renouvellement des acteurs. Néanmoins, dès 1945, le Dr. Meillon, dans son rapport sur les SI, rappelle que le tourisme est « l'œuvre assidue et souvent courageuse de tous nos groupements réunis en un faisceau solidaire autour des dirigeants des SI, de leurs fédérations et de l'union nationale de ces fédérations. ${ }^{37}$ Ainsi, malgré leurs difficultés, leurs limites et leurs échecs, les actions lancées par les SI, souvent novatrices, sont progressivement reconnues. 


\section{Développer les pratiques touristiques} dans la création de nouveaux métiers et de nouvelles pratiques. C'est par exemple le cas du développement du tourisme hivernal et de l'espace montagnard, avec la création du métier de moniteur de ski. Le développement de la station Val d'Isère en offre l'exemple avec la création d'une école de ski (1934-1935). Grâce à Charles Diébold, les moniteurs de ski ont pu être formés afin de sécuriser ce sport (Travers, 2003). Cette personnalité a d'ailleurs poursuivi son initiative au sein du comité régional de Savoie qui, dès 1948, allie les personnalités des SI et des OT locaux afin d'élaborer une politique touristique des sommets plus cohérente ${ }^{38}$. On retrouve des initiatives semblables du côté de l'espace rural, nouveau territoire à conquérir pour dynamiser le tissu économique local. Ici, ce sont les Logis et les Gîtes ruraux qui héritent de l'engagement des SI. En effet, Raymond Julien-Pagès, président de l'office du tourisme du Puy et du SI de Velay, eut l'idée de rassembler les hôteliers et les restaurateurs passionnés par l'accueil afin de proposer une offre de logement nouvelle ${ }^{39}$. Ainsi est né l'hôtel Mistou à Potempeyrat (Haute-Loire) en 1948, en parallèle de la naissance des Logis de France, conférant une réputation nouvelle et confirmée à ces professionnels du tourisme. Le succès de ces nouvelles formules se confirment dans les années 1950, lorsque les vacances sont davantage prévues et organisées (Rauch, 2001).

\section{Professionnaliser leurs actions : la naissance des écoles de tourisme}

21 Si les SI ne sont pas, à eux seuls, à l'origine des écoles d'hôtellerie et de tourisme, ils s'en sont fait les promoteurs. La première école de tourisme est née à Lausanne en 1893 et a, semble-t-il, servi de modèle pour l'école hôtelière de Paris. Les SI voient dans ces établissements une opportunité de développer le tourisme, en ayant la garantie d'obtenir un personnel de qualité. En France, la première école ouvre à Thonon-lesBains, où le thermalisme réclame un personnel qualifié. La deuxième école française date de 1914 : c'est l'école de commerce et d'industrie hôtelière inaugurée à Nice en 1916 au sein de la Villa Guiglia ${ }^{40}$ (Bottaro, 2013) (cf. illustration 4). À travers cette institution, les acteurs touristiques, privés et publics, œuvrent ensemble à l'élaboration d'une formation complète destinée à créer de nouveaux métiers touristiques ou à appuyer les compétences d'activités existantes. Centré sur la pratique hôtelière, l'enseignement comporte d'autres matières, comme la culture générale ou la sténodactylographie, auxquelles s'ajoutent à partir des années 1920 la géographie touristique, l'industrie régionale ou les ateliers-écoles, favorisés par la loi Walter-Paulin de 1937 sur l'apprentissage artisanal (cf.illustration 5). Ce n'est qu'en 1937 que la section tourisme est mise en place, avant sa rapide disparition en 1939, permettant toutefois de se former au métier de guide-interprète. À sa réouverture en 1943, sur l'initiative de personnalités locales comme Ferdinand H. Pons (président du SI de Cannes), la gestion de l'école est confiée à un conseil d'administration dans lequel les membres de SI siègent aux côtés des délégués de la chambre de commerce et de l'hôtellerie. Les SI accueillent avec satisfaction l'apparition d'une formation professionnelle des membres de leur bureaux de renseignement qui, après deux années d'études, obtiennent le titre "d'agent du tourisme». La professionnalisation du 
tourisme en France, malgré des débuts timides, se confirme dans les années 1950 grâce au soutien du Commissariat général du tourisme. Le développement et la renommée des écoles hôtelières et $\mathrm{du}$ centre national d'enseignement touristique sont ainsi assurés, bien que les écoles de Paris, Strasbourg, Thonon-les-Bains, Grenoble, Nice, Clermont-Ferrand et Toulouse existaient déjà. Les acteurs nationaux ont ainsi poursuivi un mouvement de professionnalisation soutenu depuis longtemps par les acteurs locaux.

Illustration 4. École de commerce et d'industrie hôtelière de Nice

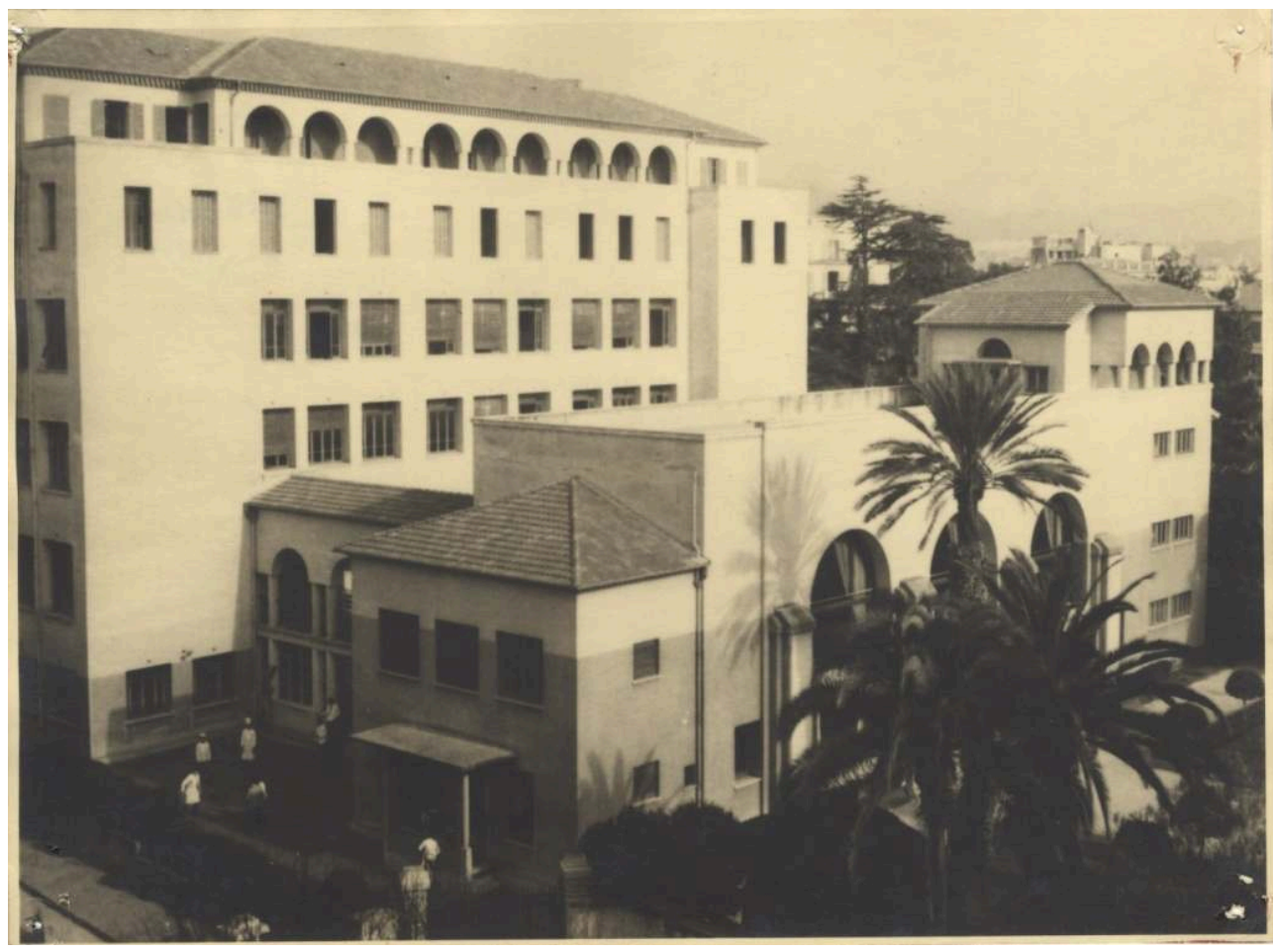

Photographie de l'École de commerce et d'industrie hôtelière niçoise, rue France, vers 1916, prêtée par Alain Callais et le CEHTAM. 
Illustration 5. Enseignements à l'école de commerce niçoise

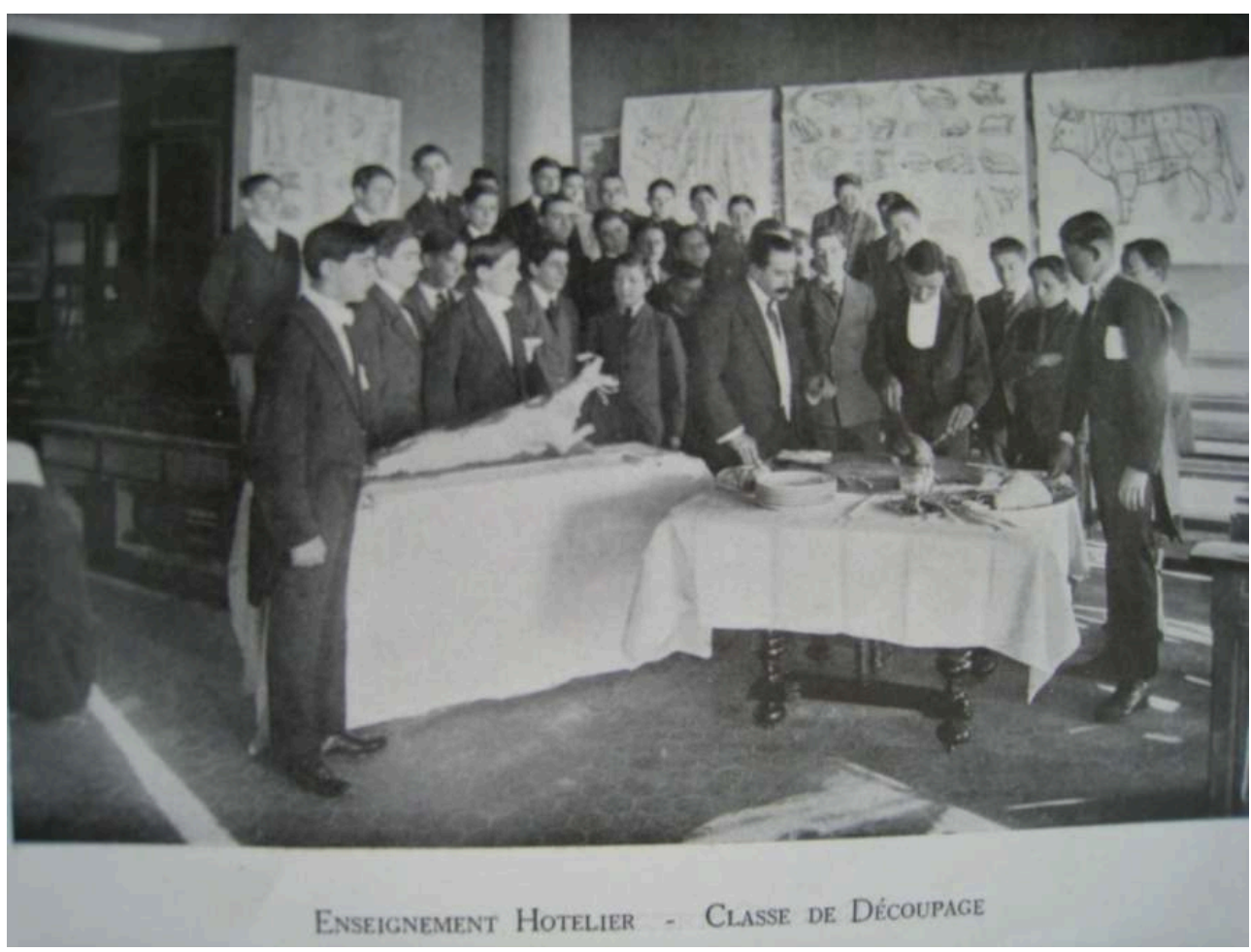

Photographie des premiers cours dispensés à l'école de commerce et d'industrie hôtelière niçoise, vers 1920, prêtée par Alain Callais et le CEHTAM.

\section{Conclusion}

Sans compter, tous les dirigeants des SI ont payé de leur temps, de leur intelligence, d'un labeur souvent acharné et qui n'allait pas sans de lourds sacrifices pour leurs intérêts particuliers [...].

Dr Meillon ${ }^{41}$

Au sein des sources mobilisées - des récits aux hommages en passant par les articles de journaux-, tous s'accordent sur la passion qui animait les administrateurs des syndicats d'initiative ; un sentiment qui s'exprimait envers le tourisme mais également de leur " petite patrie». Ces hommes, confiants tant dans leur héritage que dans leur avenir, qualifiés d'" amis des belles choses » ou d'" amoureux de leur ville ", formaient une communauté intéressée par le tourisme, vaste et disparate. Percevant l'importance de l'enjeu culturel pour l'avenir du tourisme et de l'économie française, ces « entrepreneurs du dépaysement » (Andrieux et Harismendy, 2011, p. 12) en ont fait un moteur de l'industrie touristique et ont ainsi créé une «culture touristique » (Guillet, 2011, p. 97).

Par leurs initiatives, les SI ont en effet fait prendre conscience aux autorités de l'importance de l'échelon local, ils ont tissé des réseaux touristiques à divers échelons et se sont impliqués dans le développement des politiques festives, hygiéniques ou d'embellissement de leur ville. S'agissant de l'activité touristique, ils ont contribué à révéler les enjeux de cette activité économique, ses différentes possibilités à travers les diverses pratiques possibles, ainsi que l'intérêt à créer des structures comme les Logis, les écoles de ski ou les maisons culturelles. De même, les SI ont contribué à répondre 
aux besoins de nombreux territoires en étudiant les tendances touristiques et ont créé de nouveaux métiers, comme celui de moniteur de ski, bien qu'ils aient aussi renforcé par ce biais la saisonnalité de ces nouveaux emplois.

Toutefois, comme le dit Philippe Violier, «la mutation d'un système à l'autre ne se produit pas à un moment bien identifié, voire à une date précise, mais se comprend par la mise en relation des faits dont les significations s'éclairent les unes les autres » (Violier, 2016). Si l'industrie du tourisme s'est construite sur un temps long, fait de modernisations techniques, d'évolution des représentations, de nouveaux désirs d'évasion, d'échanges culturels, il ne faut pas oublier le rôle des acteurs touristiques locaux qui ont œuvré à la cohérence de l'ensemble. Ainsi, en quatre-vingt ans d'existence, les SI ont permis le maintien d'un tissu économique local dynamique grâce au renforcement de l'identité territoriale, la création d'une réputation touristique et une meilleure accessibilité territoriale. Leur dynamisme publicitaire a également permis le développement des logos touristiques, dont les marques de territoire semblent aujourd'hui les héritières. Enfin, comprenant les nouveaux enjeux liés à la modernité, les SI ont très tôt soutenu la création des écoles de tourisme, favorisant la professionnalisation du tourisme. En retour, cette mobilisation économique, culturelle et touristique a fédéré les initiatives en faveur d'un territoire et de son identité, permettant leur appropriation tant par les habitants que par les touristes.

La professionnalisation des SI, devenus OT, est marquée par le tissage de réseaux solides, ce qui n'est pas sans rappeler d'autres entreprises comme celle des nageurs du Racing Club (Réau, 2011). En cherchant à améliorer les structures locales pour accueillir les touristes, à valoriser l'apport culturel du territoire à partir de leur propre héritage et à utiliser "le voyage pour exister professionnellement " (Réau, 2011, p. 223), les SI ont renforcé leur légitimité par leur professionnalisation mais ont également contribué à élargir les compétences et les métiers de l'industrie touristique en influençant à leur tour la professionnalisation des enseignements supérieurs par l'intégration des études touristiques dans les années $1970^{42}$. Aujourd'hui, les OT, à la suite des syndicats, deviennent à la fois les artisans et les victimes de cette "troisième révolution touristique " (Violier, 2016), dont la recomposition des acteurs, l'émergence de nouveaux désirs, le renouvellement du public et l'apparition de nouveaux outils mériteraient une nouvelle étude.

\section{BIBLIOGRAPHIE}

Jean-Yves ANDRIEUX et Patrick HARISMENDY (dir.), Initiateurs et entrepreneurs du tourisme (1850-1950), PUR, 2011.

Catherine BERTHO-LAVENIR, La roue et le stylo, Odile Jacob, 1999.

Catherine BERTHO-LAVENIR, « Le TCF et la politique de protection des sites au début du siècle dans le Morbihan », dans Nathalie RICHARD et Yveline PALLIER (dir.), Cent ans de tourisme en Bretagne (1840-1940), Apogée, 1996. 
Alain BOTTARo, «L'évolution de l'enseignement hôtelier, l'exemple niçois (1914-1950) », Recherches régionales, n² 203, 2013 [https://www.departement06.fr/documents/Import/decouvrir-les-am/ recherchesregionales203_14.pdf].

Alain CORBIN, L'avènement des loisirs (1850-1960), coll. « Champs Histoire », Flammarion, 1995.

Geneviève FRAISSE et Michelle PERROT (dir)., Tome IV : le XIXe siècle, dans Georges DUBY et Michelle PERROT (dir.), Histoires des femmes en Occident, coll. « Tempus ", Académique Perrin Éditions, 2002.

Franck, « Achille Lignon », Rues de Lyon, 2016 [https://www.ruesdelyon.net/allee/724-alleeachille-lignon.html].

Paul Goujon, Cent ans de tourisme en France, Le Cherche Midi, 1989.

François GUILLET, « La construction de la Normandie en tant qu'objet touristique pendant la première moitié du XIX ${ }^{\mathrm{e}}$ siècle », dans Jean-Yves ANDRIEUX et Patrick HARISMENDY (dir.), Initiateurs et entrepreneurs du tourisme (1850-1950), PUR, 2011.

Jean-Michel HOERNER et Catherine SICART, La science du tourisme : précis franco-anglais de tourismologie, Balzac Éditeur, 2003.

Bertrand LARIQUE, « Les débuts et les déboires de l'organisation officielle du tourisme en France : l'expérience malheureuse de l'office national du tourisme (1910-1935) », Entreprises et Histoire, $n^{\circ} 47,2007$.

Bertrand LARIQUE, L'économie du tourisme en France des années 1890 à la veille de la Seconde Guerre mondiale : organisation et développement d'un secteur socio-économique, thèse d'histoire, Université Michel de Montaigne-Bordeaux III, 2006.

Stefano LEVATI, « Les notables napoléoniens : du cas français à celui italien », Rives Méditerranéennes, $\mathrm{n}^{\circ}$ 32-33, 2009 [https://journals.openedition.org/rives/2969].

Christine MANIGAND, Henry de Jouvenel, Presses Universitaires de Limoges, 2000.

Nabila OULEBSIR, « Du voyage pittoresque au tourisme patrimonial : l'action des syndicats d'initiative nord-africains, ou l'invention d'une Méditerranée moderne », dans Jean-Yves ANDRIEUX et Patrick HARISMENDY (dir.), Initiateurs et entrepreneurs du tourisme (1850-1950), PUR, 2011.

Yves PALLIER, « Le Breton, le touriste et le pittoresque : la revue Bretagne (1933-1937) de l'exposition universelle aux musées folkloristes ", dans Nathalie RICHARD et Yveline PALLIER (dir.), Cent ans du tourisme en Bretagne (1840-1940), Apogée, 1996.

Olivier PoujoL, « Une association centenaire : le Club Cévenol », 2012 [www.club.cevenol.org/Fr/ histoire.php].

André RAUCH, Vacances en France de 1830 à nos jours, coll. « Pluriel », Hachette Littératures, réédition 2001.

Bertrand RÉAU, Les Français et les vacances, CNRS Éditions, 2011.

Alain REY, Dictionnaire historique de la langue française, T.2, Le Robert, 2012.

François RoTH, Léon Goulette, le premier directeur de l'Est Républicain, 2004 [http://devoo.xyz/ academiestanislas/images/Publications/TomeXVIII/TomeXVIII-Roth.pdf].

Catherine SICART, « Les découvreurs du tourisme aux sources de la réflexion scientifique sur le tourisme ", Les cahiers internationaux du tourisme, $\mathrm{n}^{\circ} 1,2008$. 
Alice TRAVERS, Politique et représentations de la montagne sous Vichy. La montagne éducatrice (1940-1944), L'Harmattan, 2003.

Vieux Marseille, «Inauguration de la rue Marius Dubois », actualités du comité du VieuxMarseille, [http://comitevieuxmarseille.eklablog.com/inauguration-de-la-rue-marius-duboisa5916815].

Philippe VIOLIER, « La troisième révolution touristique », Mondes de tourisme, Hors-série, 2016

[https://journals.openedition.org /tourisme/1256].

\section{NOTES}

1. En effet, dès le début du XX ${ }^{\mathrm{e}}$ siècle, les études se multiplient, dont celle de Picard (1911) ou de Stadner (1917), révélant le poids du tourisme sur les économies locales.

2. Cette thèse a été soutenue en 2015 à Paris I sous la direction de Pascal ORY.

3. 364 syndicats d'initiative existent encore aujourd'hui, contre 2278 OT.

4. Réflexion menée par Jean-Marie MIOSSEC, cité dans J-M HOERNER et C. SICART, La science du tourisme : précis franco-anglais de tourismologie, Balzac Éditeur, 2003, p. 84.

5. En effet, les sources provenant des SI sont disparates, inégales, parfois inexistantes.

6. Par «élites culturelles» nous entendons l'ensemble des personnalités intéressées par la culture sous toutes ses formes et dont les réseaux valorisent l'identité du territoire, par la publicité et l'animation des lieux. Ce groupe n'est pas homogène même si des tendances émergent à certaines époques ( $c f$. illustration 1$)$.

7. Voir Olivier POUjoL, "Une association centenaire: le Club Cévenol », 2012 [www.club.cevenol.org/Fr/histoire.php]. Paul Arnal (1871-1950), né en Lozère dans une famille d'artisans boulangers, fut bachelier en théologie puis pasteur à Uzès entre 1910 et 1937. Il a fondé le Club Cévenol à Florac le 18 septembre 1864.

8. Archives départementales des Bouches-du-Rhône (ADBDR), 212W50, lettre adressée au président du SI du Queyras, 14 mai 1949.

9. Marcel Bellon, maire d'Aiguilles-en-Queyras et directeur du SI, a créé cette récompense et l'a proposée au Commissariat général du tourisme. Cette création a été soutenue par l'un de ses amis, Maurice Petsche, alors ministre des Finances.

10. ADBDR, 212W50, "Les SI s'adaptent aux exigences modernes du tourisme», coupure de presse, s.l.n.d.

11. Franck, « Achille Lignon », Rues de Lyon, 2016 [https://www.ruesdelyon.net/allee/724-alleeachille-lignon.html].

12. Archives départementales d'Isère (ADI), 100M3/2, membres du SI de Grenoble, 1904-1905.

13. Archives municipales de Bollène (AMB), $4 \mathrm{R} 9$, délibérations du conseil d'administration du SI, le 5 juillet 1930.

14. ADBDR, 212W49, lettre adressée au commissaire général du tourisme, 27 juillet 1949. Malheureusement, nous n'avons pas de plus amples informations sur le SI d'Agay.

15. Brochure touristique sur le SI d'arrondissement d'Albertville envoyée par l'office du tourisme de la ville.

16. ADBDR, 212W52, assemblée générale du SI du Queyras, 29 août 1955.

17. Archives municipales de Montbéliard (AMMt), 62S2, "Au congrès des SI de Franche-Comté ", s.l.n.d.

18. ADI, 4332W418, "Bilan de l'action du SI à son assemblée générale », Le Dauphiné Libéré, juin 1961. 
19. Le terme est utilisé ici dans le sens employé depuis le XVII ${ }^{\mathrm{e}}$ siècle et qui « désigne une vive affection que l'on a pour quelque chose » (Rey, 2012). Un sentiment si envahissant qu'il exige un engagement inconditionnel de la part des membres des SI.

20. ADI, 41J1, M. Perret, secrétaire général du CRT de Grenoble, "L'organisation actuelle du tourisme en France ou la grande pitié des SI », causerie Radio Alpes-Grenoble, 7 avril 1948 à $19 \mathrm{~h} 15$.

21. ADI, 153M6, "Les SI, leur situation en 1914 : actuelle et dans l'avenir ", bulletin de la fédération des SI, 1914.

22. Le groupe parlementaire du tourisme est présidé, dès 1913, par Antoine Borrel et ce jusqu'en 1931.

23. Archives municipales de Vichy (AMV), journal La Tribune, le 12 octobre 1964. Ces qualités expliquent la réélection du président du SI de Vichy, Robert Mathieu.

24. $A M B, 4 R 9$, hommage à Alfred Trouillet, s.l.n.d.

25. ADBDR, 212W40, Jacques VARAGE, «Le SI de Provence : une section aixoise ", Le Mémorial d'Aix, $1903, n^{\circ} 92,66^{\text {e }}$ année.

26. Octave-Louis Aubert était également président de la chambre de commerce et de l'industrie de Saint-Brieuc.

27. ADI, 4332W418, Henri Morin, Bilan sur le tourisme français, 1965.

28. AMMt, 62S2, Dr Maulini, Première encyclique touristique, 6 octobre 1963.

29. Archives municipales de Tours (AMT), 3R446, convention entre la municipalité et le SI de Tours, décembre 1966.

30. Vieux Marseille, «Inauguration de la rue Marius Dubois ", actualités du comité du VieuxMarseille [http://comitevieuxmarseille.eklablog.com/inauguration-de-la-rue-marius-duboisa5916815].

31. Archives municipales d'Épinal (AME), 3Rb, liste des membres du SI de Nancy et des Vosges en 1903.

32. AMB, 4R9, lettre du président du SI adressée au maire, 23 octobre 1941 ; rapport du SI de Bollène, 1941, par le Commandant Trouillet et Jane Raoux.

33. AMB, 4R9, assemblée générale du SI, 1950.

34. AMB, $4 \mathrm{R} 9$, compte-rendu du conseil d'administration des SI du Vaucluse, n.d.

35. Archives nationales (AN), F12 12279, A-E Meillon, « L'œuvre des SI "ESSI" », s.l.n.d.

36. Archives municipales de Vichy (AMV), coupures de presse du journal La Montagne entre 1955 et 1965 .

37. AN, F12 12279, A-E Meillon, «L'œuvre des SI "ESSI" ", s.l.n.d. A-E Meillon est à l'époque le président de la Confédération pyrénéenne touristique, thermale et climatique.

38. Les initiatives sont proposées et explorées par Edmond Desailloud et Pierre Fallion aux côtés de Charles Diébold.

39. AMV, «La volonté de la fédération des SI d'Auvergne en faveur de l'expansion touristique de la région ", La Tribune, 1963.

40. La villa Guiglia, qui abritait autrefois l'école de commerce et d'industrie hôtelière de Nice, héberge de nos jours le Centre universitaire méditerranéen.

41. AN, F12 12279, A-E Meillon, « L'œuvre des SI "ESSI" », s.l.n.d.

42. Voir le travail mené par Hugues SERAPHIN, L'enseignement du tourisme en France et au RoyaumeUni. Histoire. Comparaisons, analyses et perspectives, coll. "Touristica Nova », éditions Publibook, 2012. 


\section{RÉSUMÉS}

En France, les réseaux touristiques ont été formés par l'action dynamique des associations touristiques, dont les syndicats d'initiative (SI) font partie. Ces structures locales, regroupant toutes les professions intéressées par le tourisme, sont issues d'une démarche novatrice apparue en 1889 à Grenoble, avec la naissance du premier syndicat d'initiative français. Destinés à l'accueil et au renseignement des touristes, les SI accomplissent également une mise en valeur touristique de leur territoire. Cependant, ces associations ont souffert d'un manque de reconnaissance et de difficultés quotidiennes qui nous poussent à nous interroger sur leur longévité. En effet, les SI ont vécu pendant quatre-vingt ans, considérés tantôt comme la base du tourisme réceptif, tantôt comme des « agence de marketing " (Oulebsir, 2011, p. 127), mais dont l'essentiel des actions au sein des réseaux touristiques reste méconnu. Il s'agit, ici, d'étudier l'histoire de ces SI à travers l'engagement de leurs bénévoles, passionnés tant par leur ville, leur région que leur patrie. En nous appuyant sur les sources émises par ces différents SI, bien que notre recherche ne soit pas exhaustive, nous souhaitons dresser le portrait de ce «monde multiforme " (Levati, 2009) qui place le tourisme au cœur de ses préoccupations. Les passions et les convictions personnelles engendrent une mobilisation active en faveur du territoire et aboutissent à la construction d'une culture commune. Les SI deviennent ainsi un outil de promotion et un symbole d'engagement, se faisant à la fois le réceptacle et l'émetteur de l'identité culturelle du territoire. Ces élites engagées ont perçu l'enjeu culturel, en ont fait un moteur de l'industrie touristique et ont ainsi créé une « culture touristique » (Guillet, 2011, p. 97). Aussi, à travers l'histoire de ces structures, des années 1900 à 1970, nous souhaitons montrer à quel point les SI ont été la cheville ouvrière, fragile mais pérenne, du tourisme français grâce à leurs membres, acteurs omniprésents au sein des réseaux touristiques français.

In France, tourist networks have been created by the dynamic action of tourist associations, of which tourist offices (syndicats d'initiative, SI) are part. These local structures, bringing together all professions interested by the tourism, come from an innovative approach that appeared in 1889 in Grenoble, with the birth of the first French tourist office (SI). Intended for the reception and information of tourists, the SI also carry out the touristic development of their territory. However, these associations lacked recognition and their daily difficulties lead us to wonder about their longevity. Indeed, the SI have lived for 80 years, considered sometimes as the foundation of receptive tourism, sometimes as "marketing agency", but whose essential actions within tourist networks remain unknown. Here, it is a question of studying the history of these SI through the commitment of their volunteers, passionate about their city; their region and their homeland. Although our research is not exhaustive, we wish to draw a portrait of this "multifaceted world" which places tourism at the center of its concerns, by relying on the sources emitted by these different SI. Passions and personal convictions generate active mobilization in favor of the territory and lead to the construction of a common culture. French tourist offices (SI) thus become a promotional tool and a symbol of commitment, being both the receptacle and the transmitter of the territory's cultural identity. These elites perceived the cultural issue, made it a driving force in the tourism industry, and thus created a "tourism culture". Also, throughout the history of these structures, between the years 1900 to 1970, we wish to show how much the SI were the backbone, fragile but lasting, of French tourism, thanks to their members, who were ubiquitous actors within French touristic networks. 
INDEX

Mots-clés : tourisme, syndicats d'initiative, identité, entrepreneurs, histoire culturelle

Keywords : tourism, French tourist office, identity, cultural studies

\section{AUTEUR}

JULIE MANFREDINI

Docteure en histoire contemporaine

Chercheuse associée à l'EIREST

jmanfredini2312@aol.com 